\title{
Quantification of Delayed Enhancement MR Images
}

\author{
Engin Dikici', Thomas O'Donnell ${ }^{1}$, Randolph Setser ${ }^{2}$, and Richard D. White ${ }^{2}$ \\ ${ }^{1}$ Siemens Corp Research 755 College Rd East Princeton, NJ 08540 \\ ${ }^{2}$ Cleveland Clinic Foundation 9500 Euclid Ave Cleveland, OH 44195
}

\begin{abstract}
Delayed Enhancement MR is an imaging technique by which nonviable (dead) myocardial tissues appear with increased signal intensity. The extent of non-viable tissue in the left ventricle (LV) of the heart is a direct indicator of patient survival rate. In this paper we propose a two-stage method for quantifying the extent of non-viable tissue. First, we segment the myocardium in the DEMR images. Then, we classify the myocardial pixels as corresponding to viable or non-viable tissue. Segmentation of the myocardium is challenging because we cannot reliably predict its intensity characteristics. Worse, it may be impossible to distinguish the infracted tissues from the ventricular blood pool. Therefore, we make use of MR Cine images acquired in the same session (in which the myocardium has a more predictable appearance) in order to create a prior model of the myocardial borders. Using image features in the DEMR images and this prior we are able to segment the myocardium consistently. In the second stage of processing, we employ a Support Vector Machine to distinguish viable from non-viable pixels based on training from an expert.
\end{abstract}

\section{Introduction}

Delayed enhancement magnetic resonance (DEMR) is an image acquisition technique applied typically to the left ventricle (LV), or the main pumping chamber of the heart, whereby non-viable tissue may be identified. Tissues that are non-viable will not benefit from interventions such as coronary by-pass or stent placement, etc. These actions serve to increase blood flow to a region, and while helpful for damaged regions provide no advantages once a tissue is necrotic. Therefore, we seek to distinguish non-viable heart tissue from healthy or damaged tissue so that unnecessary invasive procedures may be avoided and patients whose condition might improve from revascularization are identified.

Delayed Enhancement Magnetic Resonance (DEMR) is an image acquisition technique applied almost exclusively to the Left Ventricle (LV) whereby non-viable tissues may be discriminated. Typically in DEMR, a paramagnetic contrast agent (GdDTPA) is administered and the patient imaged after 20-30 minutes using a standard inversion recovery MR protocol. Non-viable cardiac tissues appear with increased signal intensity (see Figure 1a). It should be noted that during this 20-30 minute waiting period other MR protocols are generally acquired, specifically Cine MR, a time series over the cardiac cycle. Compared to DEMR, in Cine MR the LV myocardium appears with much more uniform texture (see Figure 1b) with which contraction of the myocardium can be visualized. 
The goal of our work is to automatically quantify the degree to which the left ventricle of an individual is non-viable in short axis DEMR images. This quantification is a two-step process. First, the left ventricle borders are segmented. Following this, tissues belonging to the left ventricle are classified.

Segmentation of the LV in DEMR images is challenging since we cannot reliably predict its intensity characteristics. Compared to segmentation of Cine MR images, in which assumptions may be made about the relative intensities of air, blood, and muscle, little can be said of the DEMR myocardium. Worse, non-viable tissues may have intensities that render it indistinguishable from the blood pool. Thus, radiologists in analyzing DEMR images often refer to a "corresponding" Cine image in which the heart wall is visible. By "corresponding" we mean a Cine image that has the most similar slice plane with respect to the heart and closest trigger time in the cardiac cycle. Note that slice planes may not be identical due to patient motion or respitory artifacts; and, the ECG time of the DEMR may not exactly match the temporal sampling of the Cine with respect to the ECG.
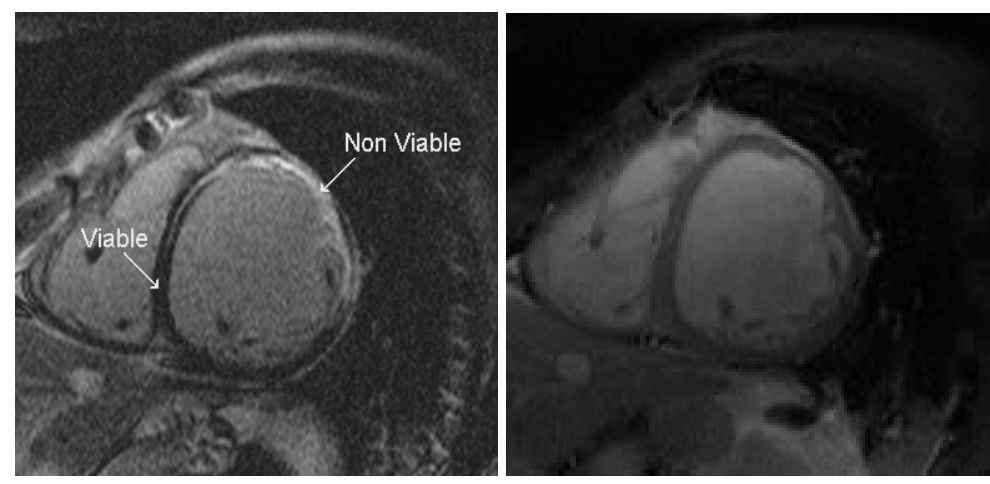

Fig. 1a. DEMR image showing viable and non-viable myocardium. Fig. 1b An MR Cine acquisition at approximately the same slice position and phase in the cardiac cycle.

In determining the myocardial border in the LV we take an approach similar to that taken by radiologists who look back and forth between the DEMR and corresponding Cine to infer the heart wall. We perform an automatic segmentation on a corresponding Cine image using the technique by Jolly [2]. While this cine segmentation is generally quite good, we make it available to the user for editing (the only manual portion of our procedure). This Cine segmentation is then employed as a prior in the segmentation of the LV in the DEMR image.

Once the LV borders in the DEMR image are identified, classification of the myocardial pixels is performed using a Support Vector Machine (SVM), a supervised machine learning technique in which the computer is taught to recognize a phenomenon given a series of examples. The SVM is trained using ground truth provided by experts at Cleveland Clinic. 


\section{Related Work}

Very little has been published on automated techniques for classifying non-viable tissues in DEMR and none, to our knowledge, on segmenting myocardial borders in these images.

The classification techniques in the literature have typically relied on either visual inspection of images [6], which precludes quantitation, or manual delineation of the non-viable region $[7,10]$, which can be time prohibitive. Semi-automatic segmentation techniques, based on the signal intensity characteristics of viable myocardium, have been used extensively in previous studies [8]. Some of these studies have defined a threshold for non-viable pixels as $>2 *$ std dev of the signal intensity of remote, viable myocardium (which was defined manually) [8]; other studies have used $3 *$ std dev as the threshold [9]. More recently, Kolipaka et al. have validated these thresholding techniques [3], demonstrating good agreement with manually thresholded images. Furthermore, this study showed that alternate thresholding methods, based on the histogram of signal intensities in the LV blood pool or the histogram of LV myocardium (including non-viable tissue) were too inconsistent for routine clinical implementation.

This work differs from our previous work [1] in that we include the segmentation of the myocardium; and, our feature space for the classification of non-viable tissues has changed. We have reduced the number of features used in the SVM as we found three of the features (e.g., thickness of the myocardium) to provide redundant information for the task. With regard to general classification using SVM, El-Naqa's work on mammogram analysis is the closest to our work [12].

To perform our segmentation of the myocardial borders we employ non-rigid registration between the DEMR and Cine images (to approximate the location of the LV in the DEMR) and between different Cine images (to interpolate the image characteristics where there is no data). Noble et. al., successfully employed Cine to Cine nonrigid registration to segment the LV in a method similar to ours [5]. They do not segment DEMR images, however, and direct Cine to DEMR registrations can only provide approximate deformation fields since the image characteristics differ so significantly.

\section{Methods}

Let $\bar{C}_{n, m}$ represent a $n \times m$ matrix of Cine images with $n$ adjacent slice positions and $m$ consecutive phases equally spaced in time. Let $\bar{V}_{n}$ represent the set of $n$ DEMR images with $n$ adjacent slice positions. For a $V_{i} \in \bar{V}_{n}$, let the corresponding cine image be $C_{i, k} \in \bar{C}_{n, m}$ such that

$$
k=\left\lfloor\frac{t_{V_{i}}-t_{C_{i, 1}}}{t_{C_{i, m}}-t_{C_{i, 1}}}\right\rfloor m
$$


where $t$ represents the ECG trigger time of the image. We apply a myocardial border detection algorithm to $C_{i, k}$ which employs a region segmentation combined with an active contour formulation [2]. The result, which may be manually edited, is $S_{C_{i, k}}(r)$, the segmentation of the corresponding Cine image. Adjacent to the corresponding cine image $C_{i, k}$ is $C_{i, k+1}$ such that

$$
t_{C_{i, k}} \leq t_{V_{i}} \leq t_{C_{i, k+1}}
$$

\subsection{Determination of Segmentation Prior}

We register $C_{i, k}$ and $C_{i, k+1}$ using a non-rigid variational approach [4]. The resulting deformation field, $U(\vec{x})$, such that

$$
C_{i, k}(\vec{x}) \mapsto C_{i, k+1}(U(\vec{x}))
$$

is linearly interpolated

$$
U^{\prime}(\vec{x})=\frac{\left(t_{V_{i}}-t_{C_{i, 1}}\right)}{\left(t_{C_{i, m}}-t_{C_{i, 1}}\right)} U(\vec{x})
$$

to calculate the deformation field at $t_{V_{i}}$. This deformation field is then applied to $S_{C_{i, k}}(r)$

$$
S_{\text {prior }}(r)=U^{\prime}\left(S_{C_{i, k}}(r)\right)
$$

to arrive at our segmentation prior, i.e., the segmentation of the cine acquisition interpolated to time $t_{V_{i}}$.

\subsection{Localization of the $\mathrm{LV}$ in the DEMR Image}

The center of the bloodpool in $V_{i}$ is computed by registering $C_{i, k}$ with $V_{i}$ resulting in the deformation field $D(\vec{x})$ such that

$$
C_{i, k}(\vec{x}) \mapsto V_{i}(D(\vec{x}))
$$

The segmentation $S_{C_{i, k}}(r)$ is then deformed by $D(\vec{x})$ to arrive at

$$
S_{\text {center }}(r)=D\left(S_{C_{i, k}}(r)\right)
$$

The centroid of $S_{\text {center }}$

$$
\vec{x}_{\text {center }}=\int_{S_{\text {center }}} S_{\text {center }}(r) d r
$$

is the position from which we start our search of the LV in $V_{i}$. Note that the deformation field is too imprecise for any other inferences. 


\subsection{Determination of the LV Borders in the DEMR Image}

We deform the prior model, $S_{\text {prior }}$, to fit to the DEMR image $V_{i}$ to maximize the probability that the resulting $S_{V_{i}}$ is the correct segmentation of $V_{i}$. In the fitting process, we apply an affine registration with five parameters: translation in the $\mathrm{x}$ and $\mathrm{y}$ dimensions $\vec{\tau}$, shearing parameters $s_{q}$ and $s_{m}$, and a scaling parameter $\omega$. The translation is bound by the distance of 10 pixels but is allowed to change without penalty. Also, the shearing is bound by \pm 60 degrees and $\pm \% 20$ scaling again without any penalty.

The scale bound, $\omega^{\prime}$, is based on the change in the size of the LV from slice level $i$ to an adjacent slice level, $i+1$ or $i-1$. The slice level with the maximal change is selected in the case of $i \neq 1, m$. We make the assumption that the change in scale due to motion artifacts, through-plane motion, etc., will not be greater than $\omega^{\prime}$.

\subsubsection{Computation of Scale Bound $\omega^{\prime}$}

For brevity we will assume the adjacent slice is $i+1$. We register $C_{i, k}$ with $C_{i+1, k}$ resulting in the deformation field $F(\vec{x})$ such that $C_{i, k}(\vec{x}) \mapsto C_{i+1, k}(F(\vec{x}))$

We then calculate the average of the deformation between the endo and epi contours of $S_{C_{i, k}}(r)$

$$
\vec{g}=\oint_{S_{C_{i, k} \text { epi }}} F(\vec{x}) d r-\oint_{S_{C_{i, k} \text { endo }}} F(\vec{x}) d r
$$

The scale bound, $\omega^{\prime}$, is then the norm of $\vec{g}, \omega^{\prime}=\|\vec{g}\|_{2}$

\subsubsection{Penalty upon Scaling}

Unlike translation, $\vec{\tau}$, a penalty is incurred on scaling. This penalty is a coefficient in our energy formulation that is maximized (to be described in Section 3.3.3). The penalty varies from 1 (no penalty) to $e^{-1}$ (maximal penalty resulting from a scaling equal to the scale bound $\omega^{\prime}$ ) and is bell shaped.

In our implementation the scale $\omega$ is iteratively increased by $1.5 \%$ from a value of 1 to $\omega^{\prime}$ (similarly decreased to $-\omega^{\prime}$ ) with the total energy formulation is evaluated at each step. Thus, at iteration $\gamma$, the scale $\omega$ is $\omega=(1.015)^{\gamma}$.

The penalty for a scale $\omega$ is based on the ratio of the iteration number, $\gamma$, and the iteration number corresponding to the maximal number of iterations, $\gamma^{\prime}$ where $\omega^{\prime}=(1.015)^{\gamma^{\prime}}$. Specifically, $e^{\frac{\gamma}{\gamma^{\prime}}}$; which may be rewritten as $e^{\frac{\log _{1.015} \omega}{\log _{1.015} \omega^{\prime}}}$. 


\subsubsection{Energy Maximization}

The energy formulation that is maximized by affine registration procedure is expressed as

$$
\begin{aligned}
& \left.S_{V_{i}}(r)=\underset{\bar{\tau}, q, m, \omega}{\operatorname{ArgMax}\left\{\left(e^{\log _{\omega^{\prime}} w}\right)\right.}\right)\left\{W_{1} \int_{S_{V_{i}}} E_{1}\left(\cos _{q} S_{m} S_{V_{i e p i}}^{\prime}(r)+\vec{\tau}\right) d r+\right. \\
& \left.\left.W_{2} \int_{S_{V_{i}}} E_{2}\left(\omega s_{q} S_{m} S_{V_{i \text { endo }}}^{\prime}(r)+\vec{\tau}\right) d r+W_{3} \underset{\omega_{q} s_{m} S_{V_{i} \text { endo }}^{\prime}(r)+\vec{\tau}}{\oint} E_{3}(x) d r\right\}\right\}
\end{aligned}
$$

where $E_{1}(\vec{x})$ and $E_{2}(\vec{x})$ are the inner and outer edge images created by applying a steering filter and detecting directional dark to bright edges and bright to dark edges respectively. Our steering filter is a modified Sobel Filter that adapts its convolution kernel by using the relative location of the convolved pixel with respect to $\vec{x}_{c e n t e r}$. $E_{3}(\vec{x})$ is the "bloodpoolness"image created by making an estimation of bloodpool's mean intensity via the intensities of the central pixels as calculated during the localization procedure.

\subsection{Classification}

We employ an SVM to perform the classification of myocardial pixels once the borders have been detected. We prefer this approach over automatic thresholding since the distribution of gray levels in the myocardium is not strictly a bimodal distribution of non-viable (bright) and viable (dark) pixels. This is due to partial voluming effects and the degree of damage.

For our kernel function of the SVM we use a Gaussian radial basis function of the form:

$$
k\left(\bar{\phi}(\vec{x}), \bar{\phi}\left(\vec{x}^{\prime}\right)\right)=e^{-\left\|\bar{\phi}(x)-\bar{\phi}\left(x^{\prime}\right)\right\|^{2} / 2 \sigma^{2}}
$$

where $\bar{\phi}$ is the vector of features. It may be shown that kernels of this form (following Mercer's conditions [11]) have corresponding optimization problems that are convex, thus lacking local minima.

To determine $\sigma$ in our kernel as well as $K$, a compromise between maximizing the margin and minimizing the number of training set errors, we employed the "leaveone-out strategy". For more details see [1]

The following three features make up $\bar{\phi}$ : The first feature, $\phi_{1}$, is the intensity of a pixel, $I_{p}$, relative to the average myocardial intensity, $\overline{I_{M}}=\frac{\sum_{p \in M} I_{p}}{\sum_{p \in M} 1}$ thus $\phi_{1}=\frac{I_{p}}{\overline{I_{M}}}$.

The second of these features is the standard deviation $\phi_{2}=\operatorname{std}\left(I_{r}\right)$ of the relative pixel intensities with respect to its next neighbors. The final feature $\phi_{3}$ is related to the image as a whole and not to a single pixel. We call this feature myocardial 
contrast and it is defined as $\phi_{3}=\frac{\overline{I_{M}}}{\bar{I}}$ i.e., the ratio of the mean myocardial intensity $\overline{I_{M}}$ and the mean image intensity $\bar{I}$ of the image.

\subsection{Image Acquisition Parameters}

Forty-five patients with known multi-vessel chronic ischemic heart disease underwent DEMR (Sonata, Siemens Medical Solutions, Erlangen, Germany) using an IR TurboFLASH sequence (FOV 300-360mm2, TE 4ms, TR 8ms, flip angle $30 \mathrm{deg}$, TI 190$470 \mathrm{~ms}$ ), approximately 20 minutes after intravenous $0.2 \mathrm{mmol} / \mathrm{kg}$ Gd-DTPA injection, with $(n=9)$ or without $(n=31)$ phase sensitive reconstruction. TrueFISP cine images (FOV 260-360mm, TE $1.5 \mathrm{msec}$, TR 25-43msec, 49-65o) were also acquired. For both types of imaging, 3 representative short-axis slices (thickness 6-10 $\mathrm{mm}$ ) were acquired at the base, mid-ventricle and apex of the left ventricle during repetitive 10-15 second breath-holds.

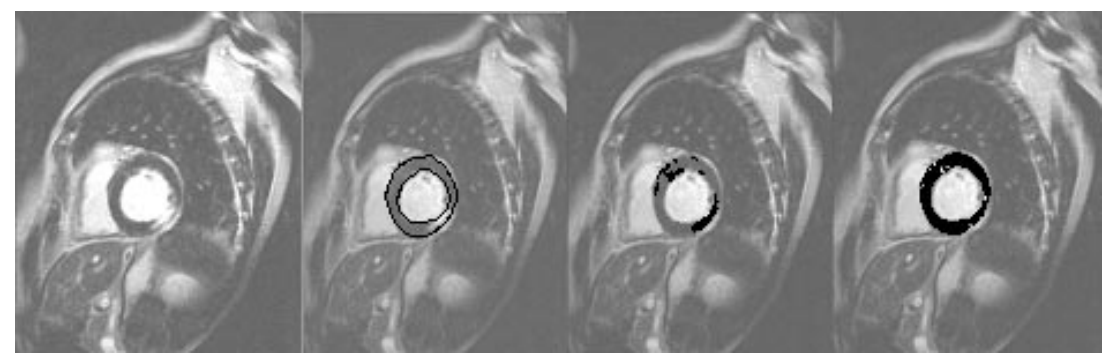

Fig. 2. Left to Right: A DEMR image. Our automatic segmentation. Our classification of nonviable tissues (black indicated non-viability). Agreement with the expert (black pixels indicate agreement; white pixels indicate disagreement).

\section{Results}

Our 45 patients were broken down in to training (31 patients) and testing (14 patients) groups. For the training group the myocardial borders were manually drawn and the pixels classified as viable or non-viable by an expert. Appropriate SVM parameters were found to be $\sigma=0.01$ and $K=20$. For the testing group, segmentations and classifications were obtained automatically. For each of the 42 (14 test patients with 3 slices levels each) DEMR images, the myocardial borders on the corresponding Cine images were automatically segmented and an expert oversaw any additional editing.

To evaluate the segmentation results, the expert delineated the ground truth myocardial borders on the DEMR images using the Argus package from Siemens. Using this ground truth we found our technique's contour pixel location error to be 1.54 pixels on average with the standard deviation of 0.39 pixels over the 42 images. 
The 42 DEMR image slices segmented above were then classified using the SVM. Our classification module achieved to $88.39 \%$ accuracy rate with a standard deviation of $6.15 \%$, sensitivity of $\% 81.34$ and specificity of $92.28 \%$. Figure 2 shows the results on one set.

\section{Conclusions}

We have presented preliminary results on the automatic segmentation and classification of non-viable tissue in DEMR images. In future work we hope to include affine transformations of the segmentation priors as well as a degree of local deformation.

\section{References}

1. O'Donnell Thomas, Ning Xu, Randolf Setser, Richard D. White. Semi-Automatic Segmentation of Non-Viable Cardiac Tissue Using Cine and Delayed Enhancement Magnetic Resonance Images. SPIE Medical Imaging 2003 Physiology and Function: Methods, Systems, and Applications, pp $242-251$.

2. M. -P. Jolly, N. Duta, and G. Funka-Lea. Segmentation of the Left Ventricle in Cardiac MR Images. Proc. ICCV, Vancouver, Canada, 2001.

3. Kolipaka A, Chatzimavroudis GP, White RD, O'Donnell TP, and Setser RM. Segmentation of Non-Viable Myocardium in Delayed Enhancement Magnetic Resonance Images. ISMRM, Toronto, CA, 2003.

4. Christophe Chefd'Hotel, Gerardo Hermosillo, and Olivier Faugeras. A Variational Approach to Multi-Modal Image Matching IEEE Workshop on Variational and Level Set Methods (VLSM'01), p.21, July 13-13, 2001.

5. Nicholas M.I. Noble, Derek L.G. Hill, Marcel Breeuwer, Julia A. Schnabel, David J. Hawkes, Frans A. Gerritsen, and Reza Razavi. Myocardial Delineation via Registration in a Polar Coordinate System. MICCAI (1) 2002: 651-658.

6. Choi, K. M., Kim, R. J. et. al.,. (2001). Transmural Extent of Acute Myocardial Infarction Predicts Long-Term Improvement in Contractile Function. Circulation 104: 1101-1107.

7. Setser RM, Bexell DG, et al. Quantitative Assessment of Myocardial Scar in Delayed Enhancement Magnetic Resonance Imaging. J Magn Reson Imaging 2003 18: 434-41.

8. R. J. Kim, D. S. Fieno, et. Al.,. Relationship of MRI delayed contrast enhancement to irreversible injury, infarct age, and contractile function. Circulation 1999; 100: 1992-2002.

9. Fieno DS, Kim RJ,et.al.,. Contrast enhanced MRI of myocardium at risk: distinction between reversible and irreversible injury throughout infarct healing. 2000;36:1985-1991.

10. Sandstede JJW, et. al.(2000). Analysis of First-Pass and Delayed Contrast-Enhancement Patterns of Dysfunctional Myocardium on MR Imaging: Use in the Prediction of Myocardial Viability. (Monographie)

11. Cristianini N, Shawe-Taylor J. An Introduction to Support Vector Machines and other kernel-based learning methods. Cambridge University Press 2000.

12. El-Naqa I,, Yongyi Yang, Miles N. Wernick, Nikolas P. Galatsanos, and Robert Nishikawa, "Support Vector Machine Learning for Detection of Microcalcififcations in Mammograms", IEEE ICIP, Rochester, NY, September 2002 